\title{
Neighborly Embedded Manifolds
}

\author{
G. Kalai · A. Wigderson
}

Received: 11 May 2007 / Revised: 19 October 2007 /

Published online: 20 February 2008

(C) Springer Science+Business Media, LLC 2008

Abstract An embedding of an $n$-dimensional manifold $M$ into $R^{d}$ is called $k$-neighborly if, for every $k$ points on the embedded manifold, there is a hyperplane $H$ in $R^{d}$ which supports the manifold precisely at these points.

Micha A. Perles (Problems presented in Oberwolfach conference on "Convexity", $1982)$ asked: What is the smallest dimension $d(k, n)$ of the ambient space in which a $k$-neighborly $n$-dimensional manifold exists?

We prove that $d(k, n) \leq 2 k(k-1) n$. Related results and open problems are discussed.

Keywords Convex bodies · Polytopes · Neighborliness · Cyclic polytopes ·

Continuous hashing

\section{Introduction}

An embedding of an $n$-dimensional (connected) manifold $M$ into $R^{d}$ is called $k$-neighborly if, for every $k$ points on the embedded manifold, there is a hyperplane $H$ in $R^{d}$ which supports the manifold precisely at these points. Namely, $H$ contains these $k$ points and all other points of the embedded manifold are in the same open half space determined by $H$.

G. Kalai is supported by NSF and BSF grants.

G. Kalai (凶)

Institute of Mathematics, The Hebrew University of Jerusalem, Jerusalem, Israel

e-mail: kalai@math.huji.ac.il

G. Kalai

Departments of Computer Science and Mathematics, Yale University, New Haven, USA

A. Wigderson

Department of Mathematics, Institute for Advanced Studies, Princeton, USA

e-mail: avi@ias.edu 
The moment curve $m(x)=\left(x, x^{2}, \ldots, x^{d}\right) \subset R^{d}$ is a [d/2]-neighborly embedding of $R^{1}$ into $R^{d}$, see, e.g., [14]. The trigonometric moment curve $(\cos t, \sin t, \cos 2 t$, $\sin 2 t, \ldots, \cos (k t), \sin (k t)) \subset R^{d}, d=2 k$ is an example of a $k$-neighborly embedding of $S^{1}$ in $R^{d}$.

Micha A. Perles [10] posed the following problem:

Problem 1 What is the smallest dimension $d(k, n)$ of the ambient space in which a $k$-neighborly $n$-dimensional manifold exists?

A $k$-neighborly embedding of any $n$ manifold $M$ is also $k$-neighborly when we restrict to a submanifold of $M$ and, in particular, to a small neighborhood of a point which is homeomorphic to $R^{n}$. Therefore for this problem we may assume that the manifold is $R^{n}$. Perles' problem is related to the problem of finding $k$-regular embeddings of manifolds into $R^{d}$. A map $f: X \rightarrow R^{d}$ is called $k$-regular if the images of every $k$ distinct points in $X$ are linearly independent. $k$-regular embeddings were studied in the context of approximation theory since the 1950 s, see e.g., $[5,7]$.

A simple dimension count shows that

$$
d(k, n) \geq(k+1) n .
$$

Indeed, let $K$ be the convex hull of a $k$-neighborly embedded $n$-manifold $M$. We have a 1-1 map from the space of all vectors $\left(\alpha_{1}, \ldots, \alpha_{k}, x^{1}, \ldots, x^{k}\right)$, where $x^{i} \in M$ for every $i$ and every $\alpha_{i}$ is a positive real number, $\sum \alpha_{i}=1$, to the boundary of $K$ which is $(d-1)$-dimensional. This implies that $d-1 \geq k n+k-1$.

There is good evidence, however, that this lower bound is never tight for $k>1$. Vassiliev [13] considered a slightly stronger definition of $k$-neighborly $C^{2}$ embeddings, where the $k$-neighborliness is stable under $C^{2}$ perturbations. Vassiliev [13] showed, by an intricate topological argument, that the analogous function $d^{\prime}(n, k)$ satisfies $d^{\prime}(k, n) \geq 2 k n-\operatorname{bin}(n)$, where $\operatorname{bin}(n)$ is the number of ones in the binary expansion of $n$. Related lower bounds for $k$-regular embeddings can be found in $[5,7]$.

A straightforward extension of the moment curve gives an upper bound for $d(k, n)$ which is exponential. For this purpose we use the embedding $\Phi_{k, n}$ defined as follows: Let $p_{0}=1, p_{1}, p_{2}, \ldots, p_{d}$ be all monomials of degree $\leq k$ in $n$ variables $x_{1}, x_{2}, \ldots, x_{n}$. Embed $R^{n}$ into $R^{d}$, by assigning to $x=\left(x_{1}, x_{2}, \ldots, x_{n}\right)$, the $d$-vector $\left(p_{1}(x), p_{2}(x), \ldots, p_{d}(x)\right)$. It is easy to see that this embedding is [k/2]-neighborly. Here is the proof: Let $x^{1}, \ldots, x^{m}$ be $m=[k / 2]$ points in $R^{n}$ and let

$$
P(x)=\left\langle x-x^{1}, x-x^{1}\right\rangle \cdot\left\langle x-x^{2}, x-x^{2}\right\rangle \cdots\left\langle x-x^{m}, x-x^{m}\right\rangle .
$$

$P(x)$ is a non-negative polynomial of $n$ variables $\left(x=\left(x_{1}, x_{2}, \ldots, x_{n}\right)\right)$ of degree $2 m$ which vanishes precisely on the points $x^{1}, \ldots, x^{m}$. Therefore, it defines a hyperplane in $R^{d}$ which supports the embedded $R^{n}$ and touches it precisely at the given points. Convex hulls of the images of finite sets $S$ of points in $R^{n}$ under the map $\Phi_{k, n}$ form an interesting class of convex polytopes that we briefly discuss in Sect. 3. However, $\Phi_{k, n}$ provides only upper bound on $d(k, n)$ which is exponential in $k$.

The main purpose of this paper is to present a simple construction showing a polynomial upper bound on $d(k, n)$. 
Theorem 2 There is a $k$-neighborly embedding of $R^{n}$ into $R^{d}$ for $d=2 k(k-1) n$.

Our construction is based on hashing and is related to natural "continuous" analogs considered by Clark, McColm, and Shekhtman [4] of standard hashing problems.

An obvious remaining challenge is to close the gap between the lower and upper bounds for $d(n, k)$. A bound of the form $O(k n)$ or even $2 k n$ may be realistic. Further problems and connections are discussed in Sect. 3. Let us mention here that 2-neighborly embeddings of $S^{3}$ into $R^{17}$ occurs naturally as faces of the "mass ball" (the convex hull of the Grassmanian $G(n, m)$ embedded into the $m$ th exterior power of $\left.R^{n}\right)$, see [2,8].

\section{Continuous Hashing and the Construction}

Call a function $g: R^{n} \rightarrow\left(R^{s}\right)^{r}, g=\left(g_{1}, \ldots, g_{r}\right)$, where each $g_{i}$ maps $R^{n}$ to $R^{s}$ $k$-universal with parameters $(n, s, r)$, if any $k$ distinct points in $R^{n}$ are mapped into $k$ distinct points in $R^{s}$ by at least one of the $g_{i} \mathrm{~s}, i \in[r](=\{1, \ldots, r\})$. Finding $k$-universal functions is a standard hashing problem; here it is unusually situated in a continuous domain, as opposed to the usual discrete one.

In order to prove Theorem 2 we require the beautiful construction of Clark, McColm, and Shekhtman's [4] $k$-universal family (consisting of linear functions) of parameters $(n, 1, r)$ for $r=n(k-1)$. Clark, McColm, and Shekhtman [4] proved a matching lower bound for smooth functions and a lower bound $r \geq[(n-1) k / 2]$. To make the paper self contained, here is a simple construction for the $k$-universal family of parameters $(n, 1, t)$ where $t=n\left(\begin{array}{l}k \\ 2\end{array}\right)+1$.

Set $t$ distinct real numbers $\alpha_{1}, \alpha_{2}, \ldots, \alpha_{t}$, and define

$$
f_{i}\left(z_{1}, z_{2}, \ldots, z_{n}\right)=\sum_{\ell=1}^{n} z_{\ell} \alpha_{i}^{\ell} .
$$

To see that this gives a universal family, consider $k$ distinct points in $R^{n}$, $z^{1}, z^{2}, \ldots, z^{k}$. For $j \in[k]$, define

$$
P_{j}(\alpha)=\sum_{j=1}^{n} z_{i}^{j} \alpha^{j}
$$

Note that $f_{i}\left(z^{j}\right)=P_{j}\left(\alpha_{i}\right)$. For every $j_{1}, j_{2} \in[k], P_{j_{1}}$ and $P_{j_{2}}$ can agree on at most $n$ points. Therefore, since $t>\left(n\left(\begin{array}{l}k \\ 2\end{array}\right)\right)$, there is $i \in[t]$ such that all $P_{j}\left(\alpha_{i}\right), j \in[k]$, are different.

Proof of Theorem 2 We will now show that the existence of a $k$-universal function of parameters $(n, s, r)$ implies that $d(n, k) \leq r \cdot d(s, k)$, since we can compose it with the $k$-neighborly solution for $R^{s}$ on each of the $r$ blocks separately. We will use this fact only for the case $s=1$ that we now explain in more detail.

Let $g$ be a $k$-universal function of parameters $(n, 1, r)$ and consider the embedding that maps a point $x=\left(x_{1}, x_{2}, \ldots, x_{n}\right)$ to the $(r \times 2 k)$ matrix $M(x)$ whose $(i, j)$-entry 
is $g_{i}(x)^{j}, i \in[r]$ and $j \in[2 k]$. First note that for every $k$ points $x^{1}, x^{2}, \ldots, x^{k}$, the matrices $M\left(x^{1}\right), M\left(x^{2}\right), \ldots, M\left(x^{k}\right)$ are linearly independent. This follows from the fact that there is a row $i$ such that $g_{i}\left(x^{\ell}\right)$ are all distinct for $\ell \in[k]$ and therefore restricted to the first $k$ columns, the $i$ th rows of the $k$ matrices correspond to the Vandermonde matrix and are linearly independent.

Next, from the neighborliness property of the moment curve it follows that for every $k$ points $x^{1}, \ldots, x^{k}$ and every $\ell \in[r]$, there is an affine functional $\rho_{\ell}$ on the space of $(2 k \times r)$ real matrices with the following properties:

(1) $\rho_{\ell}$ depends only on the entries of the $\ell$ th row.

(2) $\rho_{\ell}$ is non-negative on matrices whose $m$ th row is of the form $\left(a, a^{2}, \ldots, a^{2 k}\right)$, and vanishes on such a vector $a$ if and only if $a=\left(g_{i}^{j}\left(x^{p}\right)\right)$ for some $p \in[k]$.

A positive linear combination of $\rho_{1}, \ldots, \rho_{r}$ will vanish only for $x$ so that for every $\ell, M_{\ell}(x)$ affinely depend on $M_{\ell}\left(x^{1}\right), \ldots, M_{\ell}\left(x^{k}\right)$. This implies that for every $\ell$ there is $j$ so that $M_{\ell}(x)$ is equal to $M_{\ell}\left(x^{j}\right)$, but we showed that this is not the case unless $x=x^{j}$ for some $j$.

\section{Discussion}

We will briefly discuss Perles' question in the wider context of understanding convex hulls and order types for embedded manifolds.

The (affine) order type of a set $X$ of points in $R^{d}$ is a map from each $d+1$ ordered tuples $\left(x^{0}, \ldots, x^{d}\right)$ to $\{-1,0,1\}$ defined as the sign of the determinant of the $d+1$ by $d+1$ matrix whose $i$ th row is $\left(1, x^{i}\right)$. There is an extensive study of order types of finite sets of points and the more general combinatorial notion of oriented matroids $[3,6]$. The questions considered here can be regarded as studying the case that $X$ is a manifold or a more general topological space rather than a finite set.

Perles' question can also be regarded as a question about the convex hull of embedded $n$-dimensional manifolds in $R^{d}$. The theory of convex polytopes [14], which studies convex hulls of finite sets of points, is rather substantial, but there are only sparse results about convex hulls of embedded manifolds. An important example to keep in mind for this context is the convex hull of rank-one, positive semi-definite matrices. (For this and other related examples see $[1,2]$.) A remarkable class of embeddings of the circle to $R^{4}$ was studied by Smilansky [12].

Problem 3 What can be said about the face structure/order type of a convex embedding of $R^{1}$ and $S^{1}$ into $R^{d}$.

Embedded manifolds give rise to interesting classes of polytopes which depend on choosing $v$ points on the manifolds. The case of polytopes obtained via the embedding $\Phi_{k, n}$ is of particular interest. For example, the embedding $\Phi_{k, 2}$ gives for each configuration $C$ of $v$ points in the plane a convex polytope in $R^{\left(\begin{array}{c}k+1 \\ 2\end{array}\right)-1}$. The image of $v$ planar points $x_{1}, x_{2}, \ldots, x_{v}$ under $\Phi_{2,2}$ is a configuration of points $y_{1}, y_{2}, \ldots, y_{n}$ in $R^{5}$. The order type of the points $y_{i}$ 's records a certain "quadratic" order type of the 
original points $x_{i}$ 's. Namely, the location of $y_{6}$ with respect to the hyperplane determined by the five points $y_{1}, y_{2}, y_{3}, y_{4}, y_{5}$ is described by the location of $x_{6}$ relative to the conic described by the five points $x_{1}, x_{2}, x_{3}, x_{4}, x_{5}$.

Given a $k$-neighborly embedding of a manifold $M$ (we can assume $M=R^{n}$ ) into $R^{d}$, the convex hull of the images of every $v$ points in $M$ is a $k$-neighborly (hence also $(2 k-1)$-simplicial) polytope. Choosing points which are not in general position on $M$ may lead to interesting examples of $k$-neighborly nonsimplicial polytopes. This may be relevant to the open problem of finding a (nonsimplex) $k$-neighborly $d$-polytope (or even just a $k$-simplicial $d$-polytope) whose dual is also $k$-neighborly ( $k$-simplicial). (Here, $d$ can be arbitrary large.)

We can also consider neighborly isometric embeddings:

Problem 4 Are there $k$-neighborly isometric embeddings of $R^{r}, T^{r}$ and $S^{r}$ (equipped with the standard metric) for every $r>1$. (Here, $T^{r}$ is the $r$-dimensional torus.)

We conclude with two problems which are further away from Perles' problem but still appear to be in the same general spirit. The next problem goes back to A. Vershik:

Problem 5 Find the isometric embedding of $T^{r}$ and $S^{r}$ (equipped with the standard metric) with maximum volume of the convex hull.

For $r=1$ and $d$ even, Schoenberg [11] showed that the maximum volume of the convex hull is attained by the trigonometric moment curve.

Finally, let $K$ be an arbitrary convex body in $R^{n}$ and let $F_{r}(K)$ be the space of $r$-dimensional faces of $K$.

Conjecture 6 If all $F_{r}(K)$ are compact, then

$$
\sum_{r=0}^{d-1}(-1)^{r} \chi\left(F_{r}(K)\right)=1+(-1)^{d-1} .
$$

The case that $K$ is a convex polytope is the Euler-Poincare formula and the case that $K$ is a smooth body is just the Euler characteristic of a $(d-1)$-sphere. An extension of this conjecture to arbitrary convex bodies (giving up the compactness of the set of $k$-faces) might be possible under an appropriate definition of the Euler characteristic for the individual $F_{r}(K)$ s. Results by Mulmuley [9] may be relevant.

Acknowledgements We thank Jirka Matousek for bringing [4] to our attention and for helpful discussions. We thank Chris Connell for helpful suggestions regarding isometric embeddings.

\section{References}

1. Barvinok, A.: A Course in Convexity. Graduate Studies in Mathematics, vol. 54. American Mathematical Society, Providence (2002)

2. Barvinok, A., Blekherman, G.: Convex geometry of orbits. In: Combinatorial and Computational Geometry. MSRI Publications, vol. 52, pp. 51-77 (2005) 
3. Björner, A., Las Vergnas, M., Sturmfels, B., White, N., Ziegler, G.: Oriented Matroids. Encyclopedia of Mathematics and its Applications, vol. 46. Cambridge University Press, Cambridge (1993)

4. Clark, E.W., McColm, G.L., Shekhtman, B.: An application of spanning trees to $k$-point separating families of functions. J. Lond. Math. Soc. 58(2), 297-310 (1998)

5. Cohen, F.R., Handel, D.: $k$-regular embeddings of the plane. Proc. Am. Math. Soc. 72, 201-204 (1978)

6. Goodman, J., Pollack, R.: Allowable sequences and order types in discrete and computational geometry. In: New Trends in Discrete and Computational Geometry. Algorithms Combin., vol. 10, pp. 103134. Springer, Berlin (1993)

7. Handel, D.: 2k-regular maps on smooth manifolds. Proc. Am. Math. Soc. 124, 1609-1613 (1996)

8. Harvey, R., Morgan, F.: The faces of the Grassmannian of three-planes in $R^{7}$ (calibrated geometries on $R^{7}$ ). Invent. Math. 83, 191-228 (1986)

9. Mulmuley, K.: A generalization of Dehn-Sommerville relations to simple stratified spaces. Discrete Comput. Geom. 9, 47-55 (1993)

10. Perles, M.A.: Problems presented in Oberwolfach conference on "Convexity" (1982)

11. Schoenberg, J.: An isoperimetric inequality for closed curves convex in even-dimensional Euclidean spaces. Acta Math. 91, 143-164 (1954)

12. Smilansky, Z.: Convex hulls of generalized moment curves. Isr. J. Math. 52, 115-128 (1985)

13. Vassiliev, V.A.: On $r$-neighbourly submanifolds in $R^{M}$. Topol. Methods Nonlinear Anal. 11, 273-281 (1998)

14. Ziegler, G.: Lectures on Polytopes. Graduate Texts in Mathematics, vol. 152. Springer, New York (1995) 